\title{
Neuroendocrine tumor in the presacral region: a
} case report

\begin{abstract}
The strange presentation of lesions in the pre-sacral space and the ambiguity of its clinic make it difficult to establish its diagnosis as the first possibility. Additionally, given that several germ lines converge in this space, it is possible to find a variety of lesions, one of the rarest, without incidence registered in the literature, neuroendocrine tumors. For its diagnosis there are different types of markers, among which stands out chromogranin A, and imaging studies that allow determining the characteristics of the lesions. Regarding treatment, surgical resection remains the initial management of these tumors. The following article aims to describe the case of a 27-year-old white female patient, with no significant history, who presented pain with root characteristics predominantly in the right lower limb; after which it was taken to take images showing a tumor in the pre-sacral space, so it was necessary to carry out a surgical resection of the lesion. For its part, the immunohistochemical study revealed a tumor of neuroendocrine origin grade 2 , after which it was referred to the oncology clinic. The description of this case is considered relevant given its unusual presentation and the limited number of reports in the literature, mainly at the national level.
\end{abstract}

Keywords: retroperitoneal neoplasms, sacrococcygeal region, carcinoma, neuroendocrine, case reports
Volume 8 Issue 2 - 2020

\author{
Ronel Barbosa,' Cristina Padilla, ${ }^{2}$ Ángel \\ Rojas, ${ }^{3}$ Gennifer Barajas ${ }^{4}$ \\ 'Coloproctology Surgeon, Militar University of New Granada, \\ Colombia \\ ${ }^{2}$ General Surgeon, Sanitas University Foundation, Colombia \\ ${ }^{3}$ General Surgeon, Industrial University of Santander, Colombia \\ ${ }^{4}$ General Practitioner, Industrial University of Santander, \\ Colombia
}

Correspondence: Cristina Padilla, General Surgeon, Fundación Universitaria Sanitas, 45-16 Int 2 Apto 702, Bogota, Colombia, Tel 30 I 65850 46, Email judithpa20I4@gmail.com

Received: February 21, 2020 | Published: May 12, 2020
Abbreviations: MRI, magnetic resonance imaging; CT, computed tomography

\section{Introduction}

Pre-sacral tumors are a rare group of tumors that can be of benign or malignant origin, presenting an approximate incidence of 1:40000, documenting between $0.014-0.02 \%$ of colonoscopies. This anatomical region is limited posteriorly by the sacrum and coccyx and anteriorly by the rectum. ${ }^{1-4}$ These injuries are usually more common in the female sex with an age range that can range between $29-71$ years, with up to $66 \%$ of them being of benign origin. ${ }^{1}$ Usually there is a delay in the diagnosis of these tumors given their variable symptomatology, even so, tumors derived from enterochromaffin cells and peptidergic neurons tend to manifest clinically with pelvic pain, presenting compression of nerve roots as the first differential diagnosis. ${ }^{2,5}$ There are different publications in the world literature, however, at the national level only one study of the military hospital is registered, which describes a series of 9 cases in 25 years, none of them of neuroendocrine origin. ${ }^{6}$ In the United States from 1994 to 2009 the incidence of neuroendocrine tumors increased from 2.48 to 5.85 cases per 100,000 per year, in Europe and Asia the incidence is significantly lower with ranges ranging from 1.1 to 3.24 cases per 100,000 per year; in Colombia, a series presented in the Colombian journal of gastroenterology recorded an incidence of 5.2 cases from 1988 to 1992. Despite the above, in the presacral region they are extremely rare. ${ }^{5,7,8}$ The incomplete excision or biopsy of the lesions in the presacral space is a matter of debate since it is considered that these can recur or become infected, which is why the best tool available so far remains the MRI of the pelvis, since it allows characterize the lesion and plan the surgical intervention. ${ }^{2}$ The objective of the following publication is to describe the clinical case of a 27 -year-old female patient with a pre-sacral tumor who was brought to surgical resection with a pathology that reported a neuroendocrine tumor.

\section{Clinical case}

A 27-year-old female patient from the city of Bogotá, a systems engineering student, who consulted for a 5-year course of evolution consisting of low back pain that radiated to the predominantly right hip joint and the ipsilateral lower limb, and which had exacerbated in recent months; additionally, he reported paresthesias of the right lower limb, stools of variable consistency, and denied weight loss or fecal incontinence. No significant medical and surgical history. The clinical evaluation included the performance of resonance imaging, in which a well-defined edge oval image with a hyperintense center measuring $29 \times 25 \mathrm{~mm}$ was identified in the left pre-sacrum space, in relation to the nerve root, through the second hole left sacrum (Figure 1). Subsequently, he underwent surgical resection through the abdominal route with findings of a pre-sacrum tumor of approximately $40 \times 25 \mathrm{~mm}$ of soft consistency, located on the presacral midline at the $\mathrm{S} 2$ level, without compromising or infiltrating adjacent structures (Figure 2). Postoperative recovery was satisfactory with improvement of the referred symptoms and without neurological deficit. The initial pathology was in conclusive, suggesting differential diagnoses paraganglioma, metastasis, and less likely neoplasia of neuroendocrine origin, given the presence of a richly vascularized pseudoglandular lesion. Due to the described results, an immunohistochemistry test was requested, which reported welldifferentiated grade 2 neuroendocrine tumor with a mitotic rate less than 3 mitosis in $10 \mathrm{HPF}$ without lymphovascular invasion, with a Ki67 cell proliferation index of $12 \%$. With this diagnosis, she was referred to the clinical oncology consultation to start adjuvant therapy. 

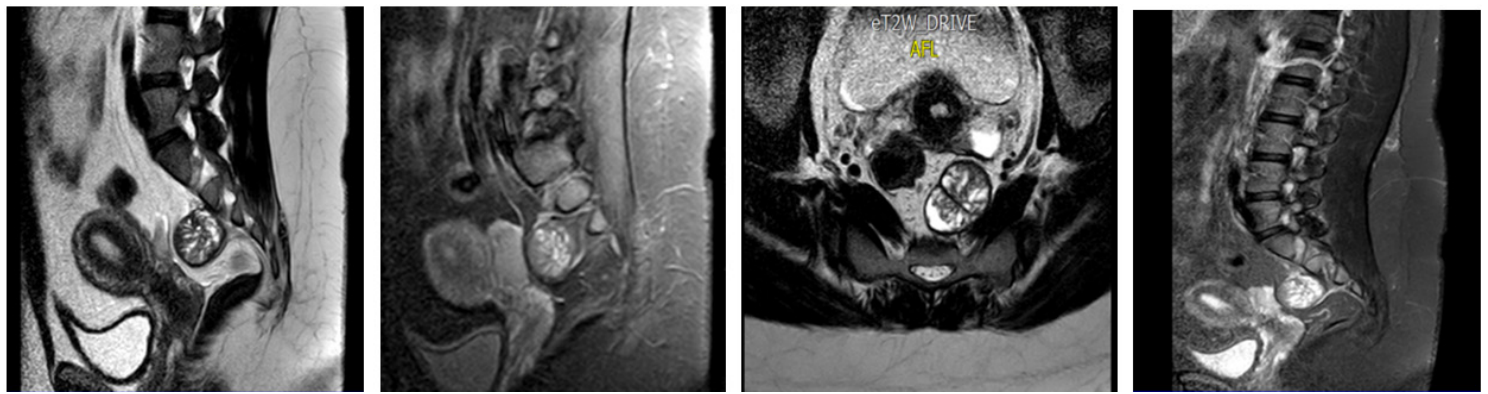

Figure I Contrasting pelvic resonance showing a hyperintense oval image in the pre-sacral space.

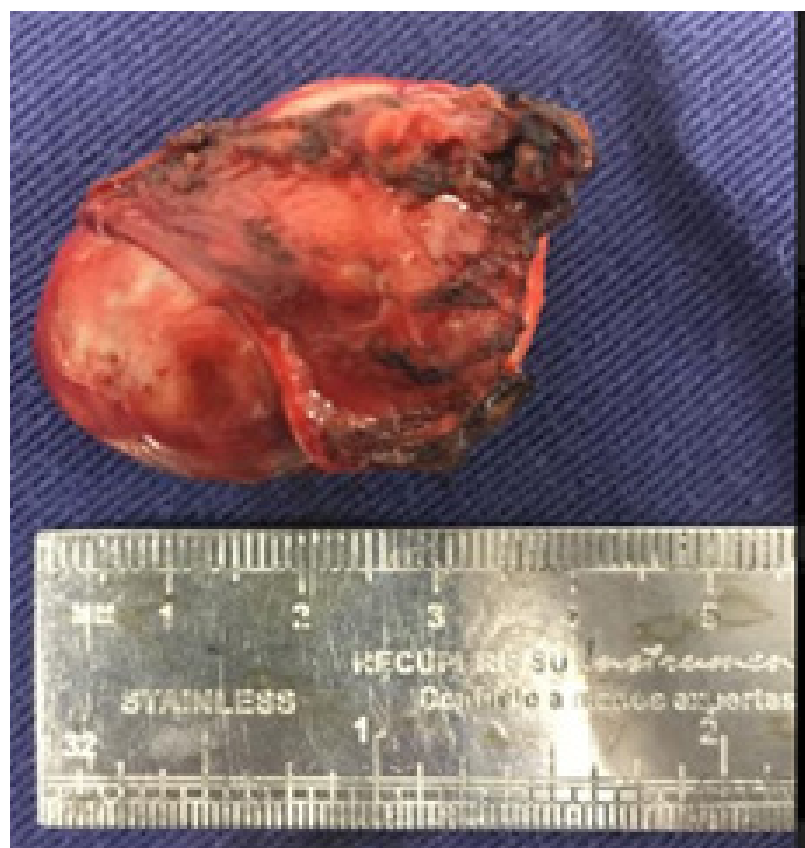

Figure 2 Photograph of the surgical piece showing a $4 \times 2 \mathrm{~cm}$ presacral lesion.

\section{Discussion}

Pre-sacral tumors were first reported by Emmerich in the mid19th century. Given its rarity, its exact percentage of presentation is currently unknown; however, some articles record an incidence of approximately 1 case in $40,000 \cdot .^{1-3,9}$ In a review of the literature, only 15 reported cases of neuroendocrine tumors of primary origin in the presacral space were found, of which up to $60 \%$ of the cases were female and the majority were associated with sacro-octogenic teratomas or cysts of the posterior intestine. ${ }^{10}$ The pre-sacral space is previously limited by the fascia of the upper $2 / 3$ of the rectum, later by the pre-sacral and endopelvic fascia, and by the ureters and iliac vessels laterally, the roof is formed by the peritoneal reflection and the floor by Waldeyer's fascia (Figure 3). ${ }^{11}$ In this region, all germ lines converge at the end of embryological development, creating a potential site of congenital, neurogenic, and bone lesions, among others, which may in turn be benign or malignant. ${ }^{12}$ One of the theories that arises about the origin of neuroendocrine tumors in the presacral region is described by Tad Kim et al., ${ }^{10}$ who suggests that these tumors derive from embryological remnants of the posterior intestine, since histopathological similarity has been found between these cells and tumors. Neuroendocrine. ${ }^{10}$ One of the most in frequent presenting lesions, in this location, are neuroendocrine tumors. It is believed that neuroendocrine cells are of neuroectodermal origin and that many of them contain secretory granules composed of biogenic amines (serotonin, histamine, dopamine, etc.) that when coming into contact with the systemic circulation trigger symptoms such as diarrhea, facial redness, among others. On the other hand, there are also non-functioning neuroendocrine tumors, which, as in the case of our patient, usually cause compression symptoms. ${ }^{13,14}$ Neuroendocrine tumors are usually more common in the gastrointestinal tract, where authors such as Arvind Dasari et al. ${ }^{15}$ recorded an increase in incidence by 6.4 times, being from 1.09 per 100,000 people in 1973 to 6.98 per 100,000 people in 2012, also noting a prevalence of $0.006 \%$ in 1993 to $0.048 \%$ in 2012 . Despite this, the percentage of presentation of these lesions in the pre-sacral space is not known exactly. ${ }^{2,5,15}$ These lesions have a variable clinic that is not only related to the release of hormones but also to their location, which is why symptoms such as digestive bleeding, jaundice, symptoms of intestinal obstruction, weight loss, among others, are usually described; on the other hand, since the root of the sacral nerves is located in the pre-sacral space, it is not strange to suppose that the symptoms of an injury in this location are usually referred to as radiculopathies or rectum anal or sexual dysfunction. ${ }^{2,13}$ The world health organization, last updated in 2017, classifies neuroendocrine tumors into three groups that are the differentiated goods, poorly differentiated and the mixed or adenoneuroendocrine. In turn, the well-differentiated are subdivided according to their proliferative activity given by the mitotic index and / or Ki67 index, which has a strong prognostic value (Figure 4). ${ }^{16}$

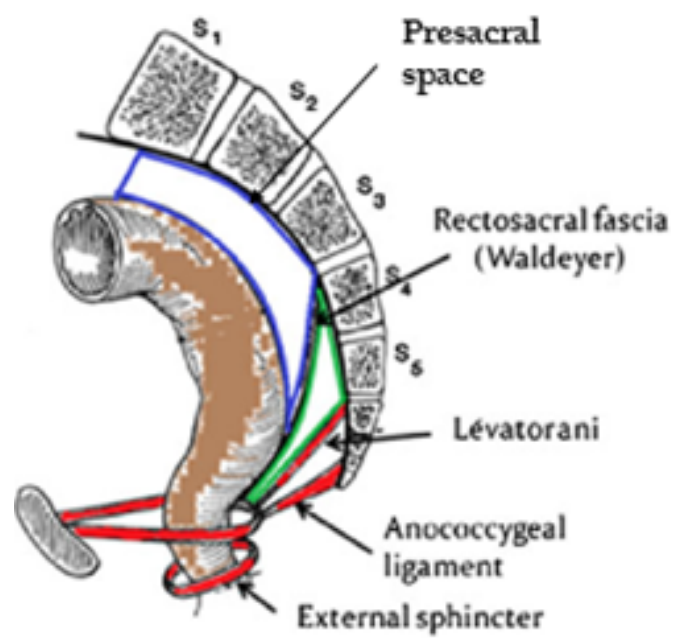

Figure 3 Excerpted from: Nedelcu M, Andreica A, Skalli M. Laparoscopic approach for retrorectal tumors. Surg Endosc. 2013;27(II):4 I77-4 I83. 


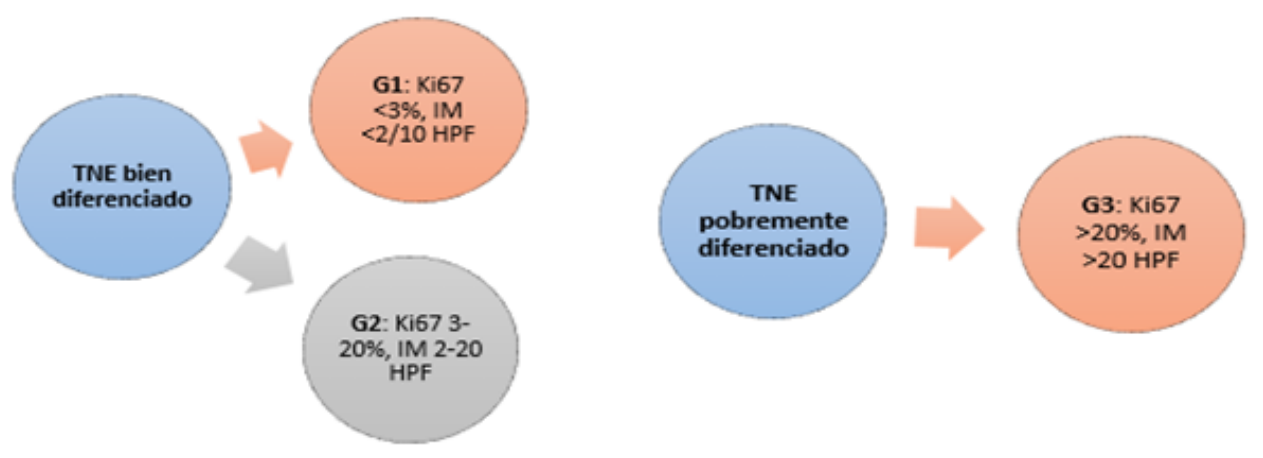

Figure 4 Adapted from WHO classification of tumors of endocrine organs, fourth edition (2017).

NET, neuroendocrine tumor; IM, mitotic index; HPF, high powerfield.

Given the rarity in the presentation of neuroendocrine tumors, in the patient of the case in question, a tumor of neurogenic origin vs a chondroma were considered as differential diagnoses. This diagnostic suspicion was raised on the one hand in relation to neuroleptic symptoms, which could suggest a lesion of the neuofibroma, ganglioneuroma or schwannoma type, and on the other hand due to the location of the lesion, with chondromas being the most common malignant tumor in space. Presacral. ${ }^{2,17,18}$ Although the first diagnostic modality is usually computed tomography (CT), nuclear magnetic resonance imaging (MRI) allows to better characterize solid cystic lesions, define the plane between the tumor and the presacral fascia, and evaluate invasion to adjacent structures, as for example in the commitment of spaces S1-S2 that defines iresecability. ${ }^{12,19}$ One of the most named controversies in the literature is the preoperative biopsy of a lesion in the pre-sacral space, this due to the high risk of complications such as infection, bleeding, neuralgia, among others, however this is recommended only in unresectable or highly aggressive, such as osteogenic sarcoma, amenable to management with radiation therapy or chemotherapy; although other authors such as Bukhetan consider that this procedure as a high risk of spreading in malignant lesions. ${ }^{2,12,20}$ Regarding treatment, most of these injuries benefit from surgical management, with an approach that will depend on the location of the tumor within the pelvis. There are three main approaches: the posterior perineal or pre-sacral, which is preferred for lesions that are below S3, the anterior or transabdominal, and the combined recommended in tumors that extend above the S3 level. ${ }^{2,17,19}$ In the case described in this publication, the transabdominal approach was used, in which the first thing dissected is the mesorectum of the anterior portion of the lesion, then separating it from the presacral fascia; before completely removing the tumor, it is necessary to control the arterial supply identifying and ligating the middle sacral artery, later it is necessary to reject the rectum to extract the surgical piece. ${ }^{18}$ Among the postoperative complications described are not only infection or the appearance of hematomas, but also the rectal fistula in 3\% of cases, as well as the loss of the voiding or defecation reflex due to nerve injury above the S2 level (mainly due to hypogastric plexus injury). ${ }^{21}$ In the case of our patient, there were no complications in the immediate postoperative period or during followup in the late postoperative period. Non-surgical treatment is reserved for chemically sensitive tumors such as B-cell lymphoma, Ewing's sarcoma, osteogenic sarcoma, or in patients with metastatic lesions, as palliative management. For its part, radiation therapy is high doses is preferred when there is incomplete excision of a chondroma or when there is local infiltration. ${ }^{19}$
Another therapy in development is immunotherapy with tyrosine kinase inhibitors, such as Imatinib, which has shown good results, increasing survival, in the management of advanced chondromas. On the other hand, epidermal growth factor inhibitors such as Cetuximab and Gefitinib have shown a good response in patients with chondromas that present local recurrence and metastasis. ${ }^{22}$ Biotherapy plays an important role in the treatment of neuroendocrine tumors, both for the control of hormonal syndromes and in tumor growth; Somatostatin analogues are usually the first line of management in most well-differentiated neuroendocrine patients or in the palliative management of carcinoid syndrome. Studies such as CLARINET and PROMID demonstrated an increase in survival at 14 and 18 months with the use of Lanreotide and Octreotide when compared to placebo. Other treatments described for the management of these tumors are Everolimus, Bevacizumab, Sunitinib, as well as cytotoxic therapy (Streptozocin, Dacarbazine, 5-Fluoracil, Capecitabine, Doxorubicin, among others) in progressive or metastatic disease..$^{13,14}$ The prognosis of pre-sacral tumors generally depends on the histological nature of the lesion and on complete surgical resection. When malignant degeneration occurs, it usually occurs in males. The recurrence rate in general is usually low with a described percentage of $7 \%$ and a survival rate that in some publications reaches $100 \%$. $^{23,24}$

\section{Conclusion}

Pre-sacral neuroendocrine tumors are lesions that have an unusual location in the pre-sacral space depending on their size and relationship with the sacral roots and adjacent organs, they expressed some type of symptomatology, which in the particular case of our patient was root-type pain imaging studies are necessary for surgical planning, and preoperative biopsy is only reserved for unresectable lesions suspected of malignancy.

Surgical treatment is usually the treatment of choice, although it involves some complications, including loss of voiding reflex, defecation and sexual function. Likewise, it is important to determine the degree of the resected neuroendocrine tumor, to determine the need for adjuvant therapy.

\section{Acknowledgments}

None.

\section{Conflicts of interest}

None of the authors declares conflicts of interest. 


\section{Funding}

None.

\section{References}

1. Canelles E, Roig JV, Cantos M, et al. Presacral tumors. Analysis of 20 surgically treated patients. Spanish Cir. 2009;85(6):371-377.

2. Champagne BJ. The ASCRS Manual of Colon and Rectal Surgery. In: Scott R Steele, Neil Hyman, Thomas E Read, editors. Diseases of the Colon \& Rectum, Switzerland: 2010. 1712 p.

3. Jao SW, Beart RW Jr, Spencer RJ, et al. Retrorectal tumors. Mayo clinic experience, 1960-1979. Dis Colon Rectum. 1985;28(9):644-652.

4. Li GD, Chen K, Fu D, et al. Surgical strategy for presacral tumors: analysis of 33 cases. Chin Med J (Engl). 2011;124(23):4086-4091.

5. Zhang R, Zhu Y, Huang XB, et al. Primary neuroendocrine tumor in the presacral region: a case report. World J Clin Cases. 2019;7(14):1884 1891.

6. Betancur N, Troncoso JM MC. Tumors presacros case series report Central Military Hospital. Militar University of New Granada; 2013.

7. Hallet J, Law CH, Cukier M, et al. Exploring the rising incidence of neuroendocrine tumors: a population-based analysis of epidemiology, metastatic presentation, and outcomes. Cancer. 2015;121(4):589-597.

8. Martínez CCV, Llano RC. Gastroenteropancreatic neuroendocrine tumors. Rev Colomb Gastroenterol. 2010;25(2):158-169.

9. Issa N, Fenig $\mathrm{Y}$, Aviran N, et al. Controversies in presacral tumors management. J Coloproctology (Rio de Janeiro). 2017;37(4):336-340.

10. Kim T, Grobmyer SR, Liu C, et al. Primary presacral neuroendocrine tumor associated with imperforate anus. World J Surg Oncol. 2007;5:115.

11. Saxena D, Pandey A, Bugalia RP, et al. Management of presacral tumors: our experience with posterior approach. Int J Surg Case Rep. 2015;12:3740

12. Wolpert A, Beer-Gabel M, Lifschitz O, et al. The management of presacral masses in the adult. Tech Coloproctol. 2002;6(1):43-49.
13. Wang R, Zheng-Pywell R, Chen HA, et al. Management of gastrointestinal neuroendocrine tumors. Clin Med Insights Endocrinol Diabetes. 2019;12:1-12.

14. Rorstad OP. Neuroendocrine tumors. In: Yalcin, Suayib, Öberg K, editors Endocrine Biomarkers: Clinicians and Clinical Chemists in Partnership. Berlin, Heidelberg: Springer Berlin Heidelberg; 2017:399-437.

15. Dasari A, Shen C, Halperin D, et al. Trends in the incidence, prevalence, and survival outcomes in patients with neuroendocrine tumors in the United States. JAMA Oncol. 2017;3(10):1335-1342.

16. Klöppel G. Neuroendocrine neoplasms: dichotomy, origin and classifications. Visc Med. 2017;33(5):324-330.

17. Amit Merchea EJD. Retrorectal tumors. In: Yeo CJ, editor. Shackelford's Surgery of the Alimentary Tract. 2019:2103-2116.

18. Neale JA. Retrorectal tumors. Semin Colon Rectal Surg. 2015;26(2):7383

19. Barraqué M, Filippello A, Brek A, et al. Surgical management of retrorectal tumors in the adult. $J$ Visc Surg. 2019;156(3):229-237.

20. Alharbi MB. Pre-sacral (retrorectal) abnormal tissue and tumors may be described by a new classification - a review article. Int J Surg Open. 2018;11:1-3.

21. Hsieh PC, Xu R, Sciubba DM, et al. Long-term clinical outcomes following en bloc resections for sacral chordomas and chondrosarcomas: a series of twenty consecutive patients. Spine (Phila Pa 1976). 2009;15(34):22332239 .

22. Hassan I, Wietfeldt ED. Presacral tumors: diagnosis and management. Clin Colon Rectal Surg. 2009;22(2):84-93.

23. Chêne G, Voitellier M. Benign pre-sacral teratoma and vestigial retrorectal cysts in the adult. J Chir (Paris). 2006;143(5):310-314.

24. Grandjean JP, Mantion GA, Guinier D, et al. Vestigial retrorectal cystic tumors in adults: A review of 30 cases. Gastroenterol Clin Biol. 2008;32(8-9):769-778. 